\title{
Retinocollicular Synapse Maturation and Plasticity Are Regulated by Correlated Retinal Waves
}

\author{
Ruchir D. Shah ${ }^{1,2}$ and Michael C. Crair ${ }^{1}$ \\ ${ }^{1}$ Department of Neurobiology, Yale University School of Medicine, New Haven, Connecticut 06510, and ${ }^{2}$ Graduate Program in Neuroscience, Baylor College \\ of Medicine, Houston, Texas 77030
}

\begin{abstract}
During development, spontaneous retinal waves are thought to provide an instructive signal for retinotopic map formation in the superior colliculus. In mice lacking the $\beta 2$ subunit of nicotinic ACh receptors $\left(\beta 2^{-l-}\right)$, correlated retinal waves are absent during the first postnatal week, but return during the second postnatal week. In control retinocollicular synapses, in vitro analysis reveals that AMPA/ NMDA ratios and AMPA quantal amplitudes increase during the first postnatal week while the prevalence of silent synapses decreases. In age-matched $\beta 2^{-1-}$ mice, however, these parameters remain unchanged through the first postnatal week in the absence of retinal waves, but quickly mature to control levels by the end of the second week, suggesting that the delayed onset of correlated waves is able to drive synapse maturation. To examine whether such a mechanistic relationship exists, we applied a "burst-based" plasticity protocol that mimics coincident activity during retinal waves. We find that this pattern of activation is indeed capable of inducing synaptic strengthening [long-term potentiation (LTP)] on average across genotypes early in the first postnatal week [postnatal day 3 (P3) to P4] and, interestingly, that the capacity for LTP at the end of the first week (P6-P7) is significantly greater in immature $\beta 2^{-l-}$ synapses than in mature control synapses. Together, our results suggest that retinal waves drive retinocollicular synapse maturation through a learning rule that is physiologically relevant to natural wave statistics and that these synaptic changes may serve an instructive role during retinotopic map refinement.
\end{abstract}

Key words: retinal waves; superior colliculus; activity dependent; synapse development; mouse; map refinement

\section{Introduction}

The regulation of synapse maturation and plasticity are thought to contribute to the activity-dependent formation and refinement of neural circuits (Katz and Shatz, 1996; Zhang and Poo, 2001). In the classical Hebbian model of sensory map refinement, neighboring coactive inputs are selectively strengthened and stabilized through a long-term potentiation (LTP)-like process, while uncorrelated inputs are selectively weakened and eliminated from postsynaptic targets (Constantine-Paton and Cline, 1998). This model predicts that neural activity serves an instructive rather than permissive role in circuit development, because its temporal pattern of activation contains information about the spatial location of presynaptic inputs (Crair, 1999). This information is subsequently read out by synaptic learning rules that guide changes in synaptic efficacy in response to various patterns of inputs.

At many immature synapses in the CNS, glutamatergic transmission is initially dominated by NMDA receptors (NMDARs)only, at so-called "silent synapses," which may serve as a template

Received Sept. 18, 2007; revised Nov. 19, 2007; accepted Nov. 21, 2007.

This work was supported by National Institutes of Health Grants R01 MH062639, R01 EY015788, P30 EY000785, and T32 EY07001. We gratefully acknowledge Anand Chandrasekaran and members of the Crair laboratory for insightful discussions and Ed Ruthazer for helpful comments on this manuscript.

Correspondence should be addressed to Dr. Michael C. Crair, Department of Neurobiology, Yale University School of Medicine, P.0. Box 208001, New Haven, CT 06520-8001. E-mail: michael.crair@yale.edu.

DOI:10.1523/JNEUROSCI.4276-07.2008

Copyright $\odot 2008$ Society for Neuroscience $\quad$ 0270-6474/08/280292-12\$15.00/0 on which activity can instruct functional connectivity (Isaac et al., 1995; Feldman et al., 1999). More mature synapses acquire transmission through AMPA receptors (AMPARs) over the course of normal development, and these effects can be hastened by experimentally inducing LTP (Liao et al., 1995; Durand et al., 1996; Wu et al., 1996; Isaac et al., 1997). NMDAR-dependent LTP is also observed during developmental periods in which activity can significantly affect patterns of structural connectivity (Crair and Malenka, 1995; Kirkwood et al., 1996), suggesting that activitydependent increases in synaptic strength may contribute to the stabilization of synaptic connections. Interestingly, both the prevalence of silent synapses and the capacity for LTP induction decline at a similar rate with age in the neocortex (Crair and Malenka, 1995; Kirkwood et al., 1995; Isaac et al., 1997; Rumpel et al., 1998; Franks and Isaacson, 2005). These phenomena are also regulated by neural activity, because a lack of sensory experience can delay synaptic maturation and extend the developmental window for LTP induction (Kirkwood et al., 1995, 1996; Franks and Isaacson, 2005). These results support the hypothesis that synaptic strengthening via specific learning rules can serve as a read-out of instructive information and provide a stabilization signal for developing circuits.

In the mouse superior colliculus (SC), the refinement of retinal ganglion cell (RGC) axon projections from an initially coarse arrangement to their precise, topographically appropriate target zones occurs via a process of activity-dependent competition that is thought to be mediated by spontaneous retinal waves 
(McLaughlin et al., 2003; Chandrasekaran et al., 2005). These waves consist of bursts of action potentials that sweep across the retina and produce highly correlated activity among neighboring RGCs, providing a substrate for Hebbian synaptic competition during retinotopic map formation (Torborg and Feller, 2005). In mice that lack the $\beta 2$ subunit of the nicotinic acetylcholine receptor $\left(\beta 2^{-1-}\right.$ mice $)$, correlated retinal waves that normally occur during the first postnatal week are absent, and anatomical refinement of the retinocollicular map is perturbed (McLaughlin et al., 2003; Chandrasekaran et al., 2005). During the second postnatal week, the normal switch from cholinergic to glutamatergic mediated retinal waves occurs at postnatal day 8 (P8) in $\beta 2^{-1-}$ mice, thereby providing RGCs with developmentally delayed, but still precise spatiotemporal correlations for $\sim 1$ week before eye opening (Bansal et al., 2000; Muir-Robinson et al., 2002). Although NMDAR-dependent synaptic maturation and synaptic plasticity have been well documented at SC synapses after eye opening (Shi et al., 2001; Colonnese et al., 2003; Lu and Constantine-Paton, 2004; Zhao et al., 2006), it remains unknown what synaptic changes occur during the period of spontaneous retinal wave activity, whether retinal wave activity is capable of inducing Hebbian plasticity during map refinement, and whether the capacity of retinocollicular synapses to exhibit Hebbian plasticity is regulated by neuronal activity or age.

Here, we characterize retinocollicular synapse development over the first 2 postnatal weeks and examine its dependence on correlated retinal waves using the $\beta 2^{-1-}$ mouse. We find that synaptic maturation is prevented in $\beta 2^{-1-}$ animals after the first postnatal week. These parameters return to control levels by the end of the second postnatal week, suggesting that the delayed onset of correlated activity is able to drive synaptic strengthening. Using the statistics of retinal waves, we confirm that a burst-based synaptic learning rule is indeed capable of inducing LTP at immature synapses early in development and that the capacity for LTP is prolonged at immature $\beta 2^{-/-}$synapses. Together, our results reveal that the synaptic changes that normally occur during the period of map refinement depend on spontaneous retinal waves, and support a model in which synaptic maturation and plasticity guide the activity-dependent instruction of retinocollicular mapping.

\section{Materials and Methods}

Mice lacking the $\beta 2$ subunit of nicotinic acetylcholine receptors $\left(\beta 2^{-1-}\right.$ mice) were generated in the Beaudet laboratory at Baylor College of Medicine and backcrossed at least six generations onto the C57BL/6 background. Genotypes were determined by genomic PCR using primer sequences and amplification parameters described by Xu et al. (1999). All experiments were performed blind to genotype; control mice were wildtype and heterozygous littermates of $\beta 2^{-1-}$ mice. Animals were treated in accordance with Institutional Animal Care and Use Committee, Baylor College of Medicine, and Yale University School of Medicine guidelines. All data values are presented in the text as mean \pm SEM, and differences in means were tested for significance using Student's $t$ test, unless otherwise indicated.

In vitro slice physiology. Parasagittal brain slices were prepared from $\mathrm{P} 3-\mathrm{P} 13$ pups by mounting the brain at an angle of $10-15^{\circ}$ relative to the midline and making $400 \mu \mathrm{m}$ sections that preserved the optic tract and stratum griseum superficiale (SGS) layer of the superior colliculus. Slices were cut in ice-cold artificial CSF (ACSF) saturated in $95 \% \mathrm{O}_{2}$ and $5 \%$ $\mathrm{CO}_{2}$ containing the following (in $\mathrm{mM}$ ): $124 \mathrm{NaCl}, 5 \mathrm{KCl}, 1.25 \mathrm{NaH}_{2} \mathrm{PO}_{4}$, $2 \mathrm{MgSO}_{4} 2 \mathrm{CaCl}_{2}, 26 \mathrm{NaHCO}_{3}$, and 11 glucose, $\mathrm{pH} 7.2,290-310$ mOsm. Slices were allowed to recover at room temperature in bubbled ACSF for at least $45 \mathrm{~min}$ before recording. Experiments were performed at room temperature with bubbled ACSF perfused at a rate of $2-3 \mathrm{ml} / \mathrm{min}$. Whole-cell recording electrodes $(2-5 \mathrm{~m} \Omega$ ) contained the following (in
mM): 100 cesium gluconate, $17.5 \mathrm{CsCl}, 8 \mathrm{NaCl}, 10$ HEPES, 0.2 EGTA, 4 Mg-ATP, 0.3 GTP, 7 phosphocreatine, and 10 BAPTA, pH 7.2-7.5, 290$310 \mathrm{mOsm}$. Whole-cell voltage-clamp recordings were performed on cells in the SGS and were always monitored initially at a holding potential of $-70 \mathrm{mV}$. Stimuli $(30-400 \mu \mathrm{A})$ were delivered every $10 \mathrm{~s}$ through bipolar stainless-steel stimulating electrodes (FHC, Bowdoinham, ME) placed in the optic tract or stratum opticum. Bath ACSF contained $10 \mu \mathrm{M}$ bicuculline (Tocris, Ellisville, $\mathrm{MO}$ ) to eliminate inhibitory currents for all experiments. Responses were considered monosynaptic if they exhibited short and constant latency $(3-5 \mathrm{~ms})$ that did not change with stimulus intensity. Data was collected and analyzed using software written in IgorPro (WaveMetrics, Lake Oswego, OR). Input and series resistances were measured continuously to monitor the health of the cell, and data were discarded from analysis if these parameters drifted $>20 \%$ over the course of the experiment. Only one cell per slice was used.

AMPA/NMDA ratios. Stable monosynaptic AMPA responses were recorded at $-70 \mathrm{mV}$ holding potential (bath contained $2 \mathrm{mM} \mathrm{Mg}^{2+}$ and 10 $\mu \mathrm{M}$ bicuculline). AMPA receptors were then completely blocked with $10 \mu \mathrm{M}$ 1,2,3,4-tetrahydro-6-nitro-2,3-dioxo-benzo[f] quinoxaline-7sulfonamide (NBQX), and the holding potential was switched to +40 $\mathrm{mV}$ holding potential to record NMDA receptor-mediated currents at the same stimulation strength. The amplitudes of the peak currents were measured for each stimulus, and AMPA/NMDA ratios were computed by taking the average of 20 sweeps at each holding potential.

Strontium evoked AMPA miniature events. Stable whole-cell voltageclamp recordings were established at a $-70 \mathrm{mV}$ holding potential, and extracellular $\mathrm{Ca}^{2+}(2 \mathrm{~mm})$ in the bath ACSF was replaced with $3 \mathrm{~mm}$ $\mathrm{Sr}^{2+} \cdot \mathrm{Sr}^{2+}$ desynchronizes vesicle release, allowing evoked miniature current events to be analyzed (Xu-Friedman and Regehr, 1999). Evoked miniature events were recorded in $1 \mathrm{~s}$ epochs every 5-10 s in IgorPro, and subsequently imported into Mini Analysis software (Synaptosoft, Decatur, GA). Amplitude thresholds were set at 2.5 times root-mean-square noise, and at least 100 events were used for analysis in each cell. Amplitude frequency histograms were created using 1 pA bins and were normalized to the number of events in each experiment. Cumulative probability distributions were created by integrating over the normalized frequency histograms.

Silent synapse analysis. Monosynaptic AMPA responses were evoked at $-70 \mathrm{mV}$ holding potential, and stimulus intensity was subsequently reduced to evoke a mixture of failures and successes. The holding potential was then switched to $+40 \mathrm{mV}$ at the same stimulation strength. Seventy to 120 events were recorded at each holding potential. For analysis, the amplitudes measured at each holding potential were converted into smoothened amplitude frequency histograms and normalized to the number of events at each holding potential. Successes were separated from failures using the bimodality in the distributions. If a clear bimodality was not observed, each sweep was examined individually to qualify the value as either a failure or success. After defining the number of failures, the difference in failure rate across holding potentials was then computed for each experiment by subtracting the failure rate at $+40 \mathrm{mV}$ from the failure rate at $-70 \mathrm{mV}$. Here, large differences represent lower failure rates at $+40 \mathrm{mV}$, and are therefore indicative of silent synapses. Differences in failure rates were averaged for each genotype at each age and tested for significance using Student's $t$ test.

Synaptic plasticity experiments. Slices were prepared at P2-P4 and P6$\mathrm{P} 7$, and patch-clamp recordings were obtained as described above. Whole-cell recording electrodes contained the following (in $\mathrm{mm}$ ): 100 K-gluconate, $20 \mathrm{KCl}, 5 \mathrm{NaCl}, 10 \mathrm{HEPES}, 0.5$ EGTA, 4 MgATP, $0.3 \mathrm{GTP}$, 7 phosphocreatine, $\mathrm{pH} 7.2-7.5,290-310 \mathrm{mOsm}$. After obtaining a monosynaptic AMPA-mediated EPSC at $-70 \mathrm{mV}$ holding potential in voltage clamp, a baseline was recorded at $0.1 \mathrm{~Hz}$ for $40-60$ sweeps. Stimulation was then turned off and the recording was switched to current clamp. Resting membrane potential was adjusted to between -60 and $-65 \mathrm{mV}$. A 1-s-long postsynaptic current injection was delivered via the amplifier to depolarize the membrane above threshold for spiking, which was typically $20-30 \mathrm{mV}$ from rest. If little to no spiking was observed, current injection remained at an intensity to elicit a $\sim 30 \mathrm{mV}$ tonic depolarization, typically $15-20 \mathrm{pA}$. After setting the amplitude of this current injection, presynaptic stimulation was turned back on and 
delivered at $20 \mathrm{~Hz}$ for $1 \mathrm{~s}$, simultaneously with the postsynaptic current. In some cases, the presynaptic stimulation increased the number of spikes evoked postsynaptically, but usually it did not. This pairing was repeated 10 times every $30 \mathrm{~s}$. The recording was then switched back to voltage clamp, and AMPA responses were again monitored at $-70 \mathrm{mV}$ holding potential at $0.1 \mathrm{~Hz}$. For "noncoincident" plasticity experiments, the exact same protocol was used, except that postsynaptic current injection was initiated $3 \mathrm{~s}$ after the onset of presynaptic stimulation during pairing. Input and series resistances were measured continuously to monitor the health of the cell, and data were discarded if these values drifted $>20 \%$ over the course of the experiment. Experiments were also discarded if small fluctuations in series resistance correlated with fluctuations in EPSC amplitude. For all plasticity experiments analyzed at P6$\mathrm{P} 7$, no differences in input resistance (control, $903 \pm 81 \mathrm{~m} \Omega ; \beta 2^{-/-}$, $879 \pm 72 \mathrm{~m} \Omega ; p=0.8$ ) or spike threshold (control, $-30.6 \pm 1.4 \mathrm{mV}$; $\beta 2^{-1-},-31.6 \pm 1.8 \mathrm{mV} ; p=0.7$ ) were observed between genotypes. At least $25 \mathrm{~min}$ of postpairing data were collected for each experiment (up to $45 \mathrm{~min}$ ), and plasticity was quantified by comparing the mean amplitude of the last $5 \mathrm{~min}$ of the recording to the mean amplitude of the last $5 \mathrm{~min}$ of the baseline, expressed as percentage of baseline. A paired $t$ test was used in each group to test whether LTP is induced on average. Because the data were not necessarily normally distributed, a Mann-Whitney $U$ test was used to test whether the distributions were significantly different from each other. To test whether the probability of inducing LTP differed across groups, we defined LTP as $>120 \%$ of baseline and used a $\chi^{2}$ test to compare the fraction of cells that showed LTP for each age and genotype.

\section{Results}

\section{AMPA/NMDA ratio changes}

At resting membrane potentials, glutamatergic synaptic transmission occurs primarily through AMPARs, and the experiencedependent strengthening of these synapses in numerous brain areas is known to involve an increase in AMPAR trafficking (Malinow and Malenka, 2002). A developmental increase in AMPA/ NMDA ratios has been observed at synapses in a variety of sensory systems, and has also been shown to depend on neural activity (Rumpel et al., 1998; Lu et al., 2003; Takahashi et al., 2003; Franks and Isaacson, 2005). Such an activity-dependent increase has also been observed at retinocollicular synapses after eye opening (Shi et al., 2001; Lu and Constantine-Paton, 2004), but it remains unknown what synaptic changes occur during the period of spontaneous activity. We therefore measured these ratios at retinocollicular synapses in $\beta 2^{-1-}$ mice and littermate controls to characterize their development and to test their dependence on correlated retinal waves (Fig. 1). We focused on three age groups for this study: $\mathrm{P} 3-\mathrm{P} 4$, a point early in cholinergic wave activity; $\mathrm{P} 6-\mathrm{P} 7$, a point at which control animals have had a week of correlated wave activity and map refinement is nearly complete, but when retinal waves have not yet begun in $\beta 2^{-1-}$ animals; and P12-P13, a point immediately before eye opening when $\beta 2^{-l-}$ animals have had nearly 1 week of correlated glutamate waves.

Using a retinocollicular slice preparation, we stimulated RGC axons in the optic tract and stratum opticum and performed whole-cell voltage-clamp recordings on neurons in the superficial visual layer (SGS) of the SC (supplemental Fig. 1, available at www.jneurosci.org as supplemental material). At all ages, we first isolated AMPA-mediated currents at $-70 \mathrm{mV}$ holding potential. After blocking AMPARs with NBQX, we then isolated the NMDA current at $+40 \mathrm{mV}$ and computed AMPA/NMDA ratios from the peak current amplitudes at each holding potential (Fig. 1A-C). Figure $1 D$ summarizes the developmental profile of AMPA/ NMDA ratio changes in control and $\beta 2^{-/-}$synapses. We found that, in control synapses, AMPA/NMDA ratios were low at $\mathrm{P} 3-\mathrm{P} 4$ but increased significantly by P6-P7 (control P3-P4, $0.36 \pm 0.05$;
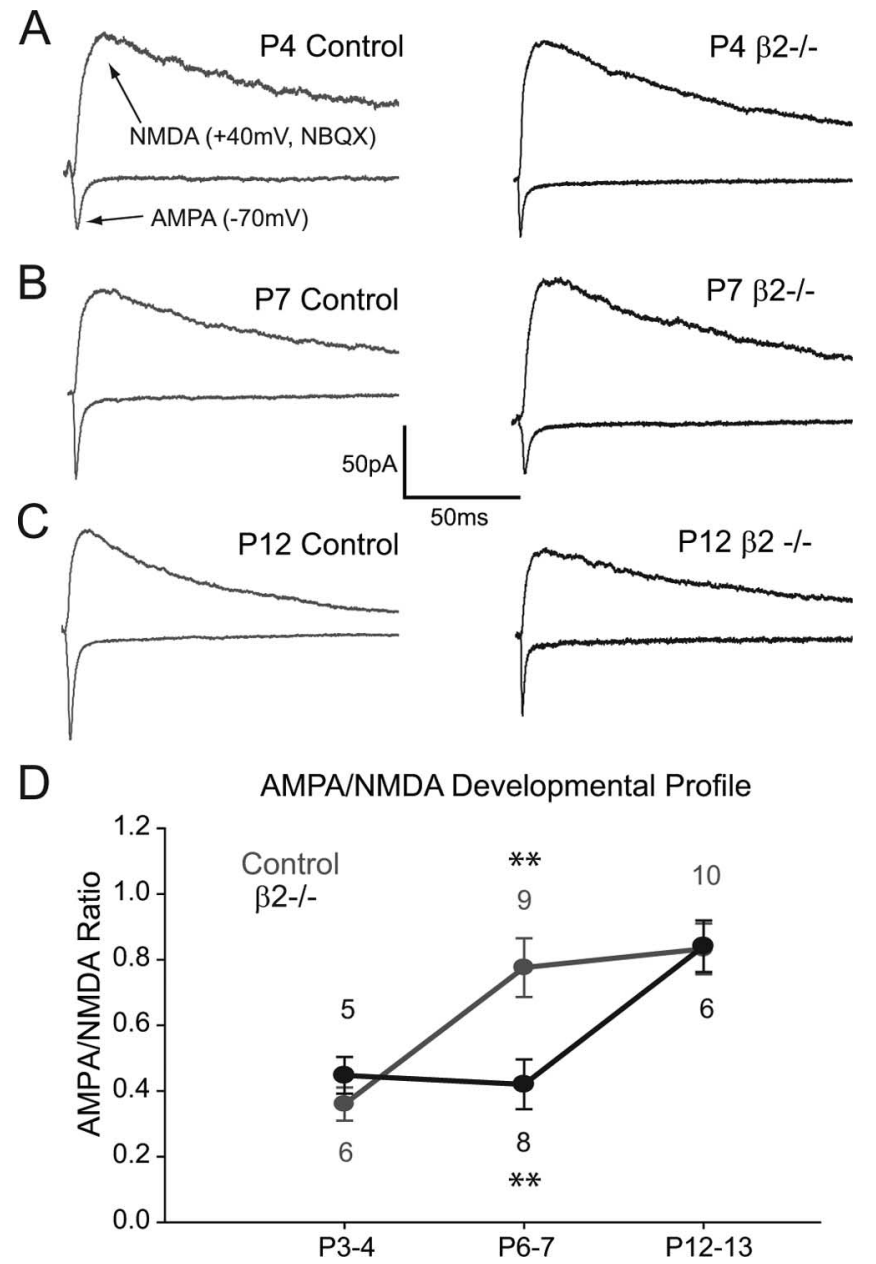

Figure 1. Developmental increase in AMPA/NMDA ratios is delayed in $32^{-/-}$retinocollicular synapses. Using extracellular stimulation, AMPA responses were evoked at $-70 \mathrm{mV}$ holding potential, and NMDA responses were isolated at $+40 \mathrm{mV}$ holding potential in $10 \mu \mathrm{m}$ NBQX. $\boldsymbol{A}$, Example AMPA/NMDA ratios for a P4 control (left) and P4 $\beta 2^{-I-}$ cell (right). $\boldsymbol{B}$, Example traces from a P7 control (left) and P7 $\beta 2^{-1-}$ (right) cell. C, Example traces from a P12 control (left) and P12 $\beta 2^{-1-}$ (right) cell. D, Summary plot depicting the developmental profile of AMPA/NMDA ratio changes in control (gray) and $\beta 2^{-1-}$ (black) synapses. Number of cells is written adjacent to each group. P6-P7 data points come from Chandrasekaran et al. (2007). In control synapses, there is a developmental increase in AMPA/NMDA ratios from P3-P4 to $\left.P 6-P 7{ }^{* *} p<0.01\right)$. This increase fails to occur in $\beta 2^{-1-}$ synapses, resulting in lower AMPA/ NMDA ratios for $\beta 2^{-1-}$ cells at $\mathrm{P} 6-\mathrm{P} 7$ relative to controls $\left({ }^{* *} p<0.01\right)$. AMPA/NMDA ratios return to control values by $\mathrm{P} 12-\mathrm{P} 13$ in $\beta 2^{-/-}$synapses $(p=0.9)$, a period that coincides with the delayed onset of correlated retinal waves. Error bars indicate SEM.

control P6-P7, $0.78 \pm 0.09 ; p<0.01)$. In $\beta 2^{-1-}$ animals, AMPA/ NMDA ratios were also low at P3-P4, but the developmental increase failed to occur by the end of the first postnatal week in the absence of correlated activity, resulting in lower ratios relative to control synapses $\left(\beta 2^{-1-} \mathrm{P} 6-\mathrm{P} 7,0.45 \pm 0.06\right.$; control P6-P7, $0.78 \pm 0.09 ; p<0.01)$. Interestingly, AMPA/NMDA ratios increased to control levels by the end of the second postnatal week in $\beta 2^{-1-}$ animals $\left(\beta 2^{-1-} \mathrm{P} 12-\mathrm{P} 13,0.84 \pm 0.08\right.$; control P12P13, $0.83 \pm 0.07 ; p=0.9$ ), suggesting that the delayed onset of correlated glutamate waves may be sufficient to drive synaptic maturation and rescue the phenotype.

\section{Development of AMPA miniature events}

The changes in AMPA/NMDA ratios that we observe could be attributable to an increase in the synaptic AMPA component, a decrease in the synaptic NMDA component, or both. To examine 
A

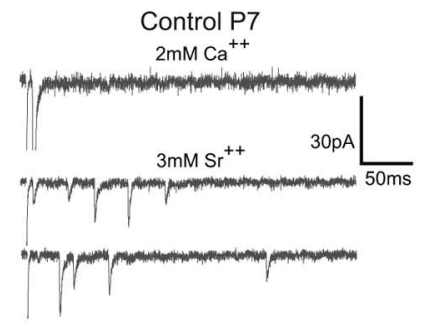

$\mathrm{B}_{1}$

Amplitude Frequency

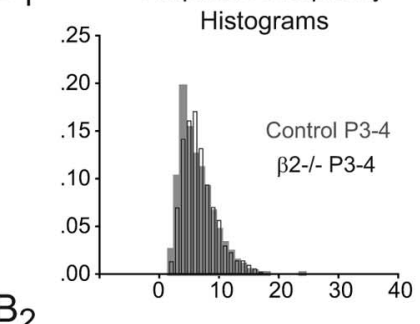

$\mathrm{B}_{2}$

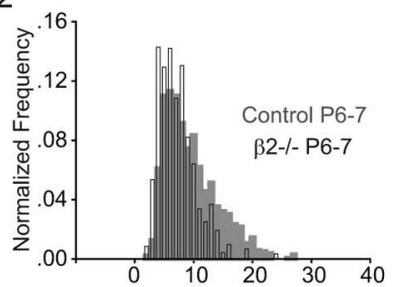

$\mathrm{B}_{3}$

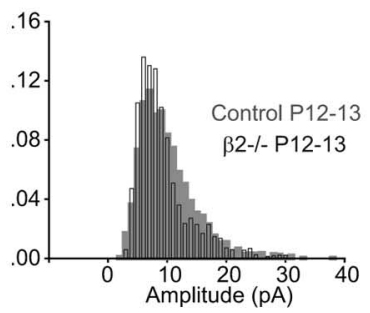

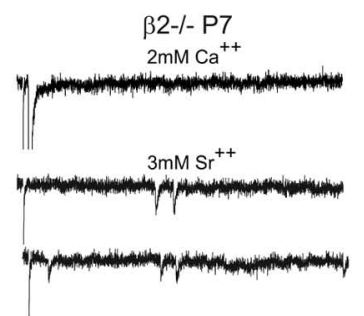

$\mathrm{C}_{1}$ Cumulative Probability Distributions

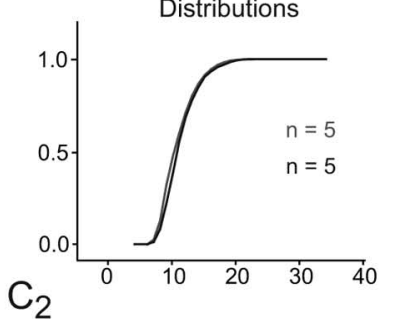

$\mathrm{C}_{2}$

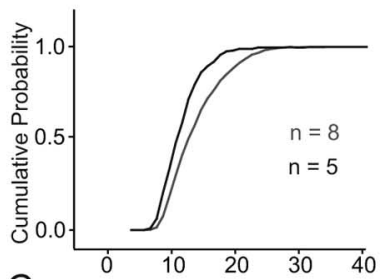

$\mathrm{C}_{3}$

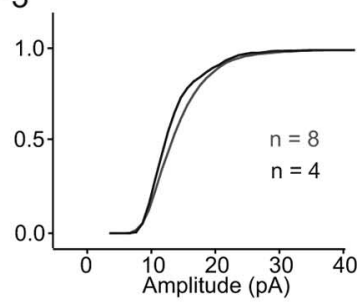

D Evoked Mini Mean Amplitude

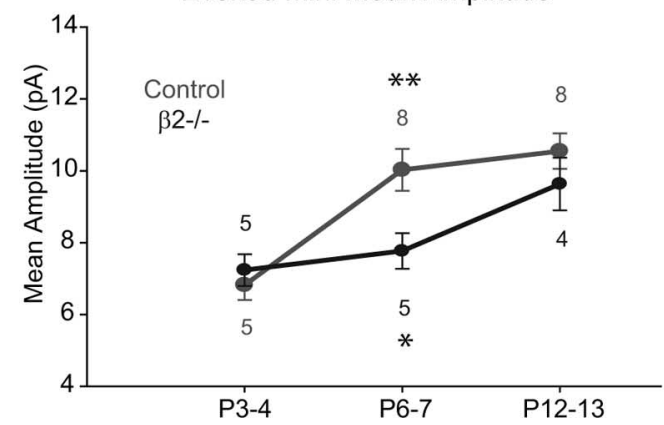

Figure 2. Developmental increase in AMPA quantal amplitudes is delayed in $\beta 2^{-/-}$retinocollicular synapses. To examine the underlying locus of the AMPA/NMDA ratio changes, we used a strontium paradigm to desynchronize vesicle release at evoked retinocollicular synapses and isolate AMPA miniature events. $\boldsymbol{A}$, Synchronous AMPA responses were initially evoked in 2 $\mathrm{mm} \mathrm{Ca}{ }^{2+}$ (top traces), and the bath solution was subsequently replaced with $3 \mathrm{~mm} \mathrm{Sr}{ }^{2+}$ (bottom traces). Examples of desynchronized AMPA events are shown for a P7 control cell (left) and a P7 $\beta 2^{-1-}$ cell (right). Note the absence of large amplitude events in the $\beta 2^{-1-}$ example. $\boldsymbol{B}$, Amplitude frequency histograms were constructed using $1 \mathrm{pA}$ bins, normalized by the number of events in each experiment, and then averaged across experiments. The normalized histograms are shown for control (filled gray bars) and $\beta 2^{-/-}$(open black bars) cells at P3-P4 $\left(\boldsymbol{B}_{1}\right), \mathrm{P} 6-\mathrm{P} 7\left(\boldsymbol{B}_{2}\right)$, and P12-P13 $\left(\boldsymbol{B}_{3}\right)$. C C Cumulative probability distributions were computed by integrating over the frequency histograms. The distributions are shown for control (gray lines) and $\beta 2^{-1-}$ (black lines) experiments at P3-P4 $\left(\boldsymbol{C}_{\mathbf{1}}\right), \mathrm{P} 6-\mathrm{P} 7\left(\boldsymbol{C}_{\mathbf{2}}\right)$, and P12-P13 $\left(\boldsymbol{C}_{3}\right)$. P6 -P7 data comes from Chandrasekaran et al. (2007). Note the leftward shift in the probability distributions for the $\beta 2^{-/-}$group at $\mathrm{P} 6-\mathrm{P} 7$ relative to the control group, indicating a greater percentage of small amplitude events. $\boldsymbol{D}$, Summary of the developmental profile for mean AMPA miniature amplitude between controls (gray) and $\beta 2^{-/-}$(black). There is a

the underlying locus of the development increase in AMPA/ NMDA ratios, we next probed changes in AMPAR-mediated miniature events at the same ages described above (Fig. 2). We first isolated AMPAR currents at $-70 \mathrm{mV}$ with $2 \mathrm{mM} \mathrm{Ca}^{2+}$ in the bath (Fig. $2 \mathrm{~A}$, top). We then replaced the extracellular $\mathrm{Ca}^{2+}$ with $3 \mathrm{mM} \mathrm{Sr}^{2+}$, which serves to desynchronize vesicle release at stimulated axon terminals. The events that we record postsynaptically are therefore events at single synapses, or miniature events (Fig. $2 \mathrm{~A}$, bottom). By measuring the peak amplitude of all the miniature events for each experiment, we constructed amplitude frequency histograms (Fig. $2 B, 1-3$ ) and cumulative probability distributions (Fig. 2C, 1-3) for all ages and genotypes. Figure $2 D$ shows the summary of the mean AMPA mini-amplitudes for control and $\beta 2^{-1-}$ synapses at each age. We find that AMPA mini-amplitudes are low for both genotypes at P3-P4 $\left(\beta 2^{-/-}\right.$ P3-P4, 7.2 \pm 0.44 ; control P3-P4, $6.8 \pm 0.42$; $p=0.5$ ), but show a significant increase in control synapses by P6-P7 (control P3$\mathrm{P} 4,6.8 \pm 0.42$; control P6-P7, $10.1 \pm 0.58 ; p<0.01$ ). Like the AMPA/NMDA ratios, this increase fails to occur in $\beta 2^{-1-}$ synapses at $\mathrm{P} 6-\mathrm{P} 7$, resulting in significantly lower values relative to controls $\left(\beta 2^{-1-} \mathrm{P} 6-\mathrm{P} 7,7.7 \pm 0.5\right.$; control P6-P7, $10.1 \pm 0.58$; $p<0.05)$. By the end of the second postnatal week, however, there is a complete rescue of the phenotype $\left(\beta 2^{-1-} \mathrm{P} 12-\mathrm{P} 13\right.$, $9.6 \pm 0.73$; control P12-P13, $10.5 \pm 0.49 ; p=0.3$. We conclude that at least part of the AMPA/NMDA ratio increase observed in control synapses is attributable to an increase in the synaptic AMPA component over the first postnatal week. This increase fails to occur in the absence of correlated retinal waves in $\beta 2^{-1-}$ animals at $\mathrm{P} 6-\mathrm{P} 7$, but is accomplished during the second postnatal week, again perhaps because of the onset of correlated glutamate waves.

\section{Prevalence of silent synapses over development}

Across numerous brain regions, another hallmark of glutamatergic synapse maturation involves the formation of "silent," or NMDAR-only, synapses early in development (Durand et al., 1996; Isaac et al., 1997; Rumpel et al., 1998). These synapses provide an important theoretical framework for understanding both the mechanisms of LTP and the activity-dependent refinement of neural circuits (Isaac et al., 1995). To examine the developmental profile of silent synapses in the retinocollicular pathway, we used a minimal stimulation assay. A representative experiment for a P7 control cell is shown in Figure $3 A-C$ and an experiment for a P7 $\beta 2^{-1-}$ cell in Figure $3 D-F$. As shown in Figure $3, A$ and $D$, we first found a stimulation strength that evoked a mixture of AMPAR-mediated successes and failures at $-70 \mathrm{mV}$. We then switched the holding potential to $+40 \mathrm{mV}$, and recorded the mixed AMPA and NMDA-mediated currents. The presence of silent synapses can be detected in this manner by comparing the failure rates at the two holding potentials, because a decrease in failures at $+40 \mathrm{mV}$ is indicative of synapses that have NMDAR-only currents and are subsequently "unmasked" at the depolarized holding potentials. We measured the amplitude of every event at $-70 \mathrm{mV}$ and $+40 \mathrm{mV}$ for each experiment and then constructed normalized frequency histograms, exam-

significant increase in AMPA mini-amplitudes in control synapses from P3-P4 to P6-P7 $\left({ }^{* *} p<0.01\right)$. Like the AMPA/NMDA ratios, this increase fails to occur in $\beta 2^{-/-}$synapses, resulting in lower mean amplitudes for $\beta 2^{-1-}$ cells at P6 $-\mathrm{P7}$ relative to controls $\left({ }^{*} p<0.05\right.$ ). AMPA miniature amplitudes also return to control values by $P 12-P 13$ in $\beta 2^{-I-}$ synapses $(p=0.3)$, suggesting that at least part of the AMPA/NMDA ratio changes are attributable to developmental increases in the synaptic AMPA component. Error bars indicate SEM. 
ples of which are shown in Figure 3, $B$ and $F$. From these histograms, we computed the failure rate at each holding potential for each experiment and plotted them as paired ladder plots for each age group (Fig. $3 G, 1-3)$. We can detect the presence of silent synapses in this manner by analyzing the difference in failure rates for each experiment $(-70 \mathrm{mV}$ minus $+40 \mathrm{mV})$, as a high difference is indicative of NMDARonly synapses. Figure $3 \mathrm{H}$ shows the developmental profile for the mean difference in failure rates across ages and genotypes. Early in development at P3-P4, we find a similarly high difference in failure rates at both $\beta 2^{-1-}$ and control synapses $\left(\beta 2^{-1-}\right.$ P3-P4, $0.21 \pm 0.03$; control P3-P4, $0.24 \pm$ $0.02 ; p=0.5)$. Over the first postnatal week, there is a significant decrease in the number of silent synapses in control animals, although some are retained (control P3-P4, $0.24 \pm 0.02$; control P6-P7, $0.06 \pm$ $0.03 ; p<0.01)$. This decrease fails to occur in $\beta 2^{-1-}$ synapses, leading to a significantly higher number of silent synapses in $\beta 2^{-/-}$animals at $\mathrm{P} 6-\mathrm{P} 7$ relative to littermate controls $\left(\beta 2^{-/-} \mathrm{P} 6-\mathrm{P} 7,0.17 \pm 0.04\right.$; control P6-P7, $0.06 \pm 0.03 ; p<0.05)$.

Together, our results suggest that, as cholinergic waves propagate through the retina, retinocollicular map refinement is accompanied by specific synaptic changes, including an increase in AMPA/NMDA ratios, an increase in AMPA quantal amplitude, and a decrease in the prevalence of silent, NMDAR-only synapses. In the absence of cholinergic waves in $\beta 2^{-/-}$animals, synaptic maturation fails to occur, and the retinocollicular network remains in an immature state. Interestingly, there is a complete rescue of the synaptic phenotype by the end of the second postnatal week in $\beta 2^{-/-}$mice and all parameters return to control levels, suggesting that the onset of correlated RGC activity via glutamate waves may provide the necessary signal for synaptic maturation. Because correlated activity is thought to induce synaptic strengthening through a Hebbian LTPlike process, we next investigated whether such a mechanistic relationship exists between retinal wave activity and synapse maturation.

\section{A burst-based learning rule can induce LTP at retinocollicular synapses}

To probe such a relationship, we examined whether the statistics of natural retinal wave activity are capable of inducing changes in retinocollicular synaptic strength. In mice, cholinergic retinal waves induce bursts of action potentials in RGCs at a spike rate of $10-20 \mathrm{~Hz}$ that last for $\sim 1-2 \mathrm{~s}$ (McLaughlin et al., 2003; Torborg et al., 2005). The properties of retinal waves thought to be essential for anatomical refinement in the LGN are the high-frequency bursting and temporal correlations among neighboring RGCs Error bars indicate SEM.
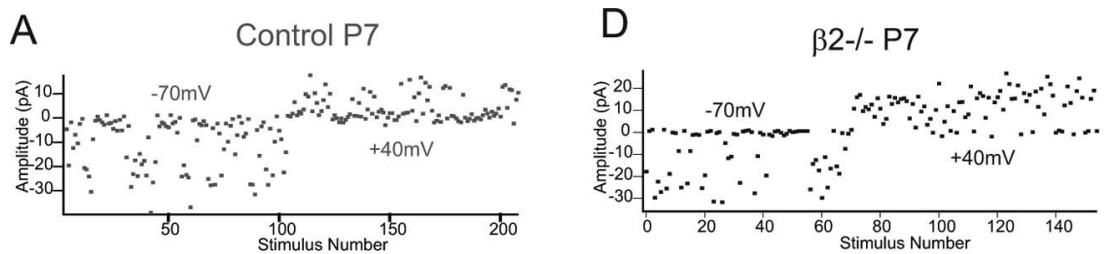

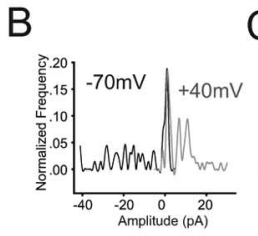

C $-70 \mathrm{mv}$
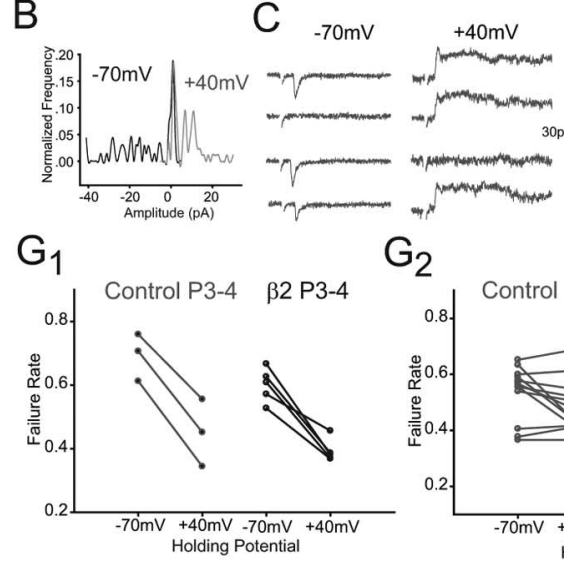

E $-70 \mathrm{mv}$

F

$\mathrm{G}_{3}$

$\mathrm{G}_{2}$

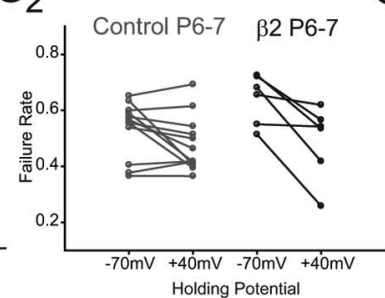

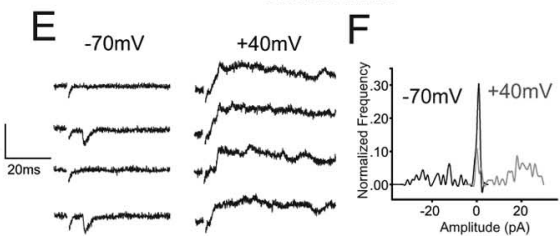

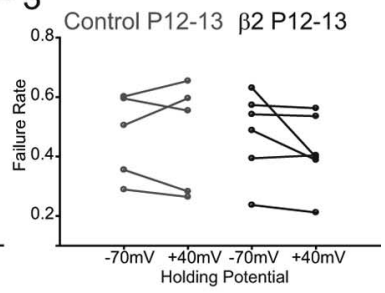

H Silent Synapse Developmental Profile

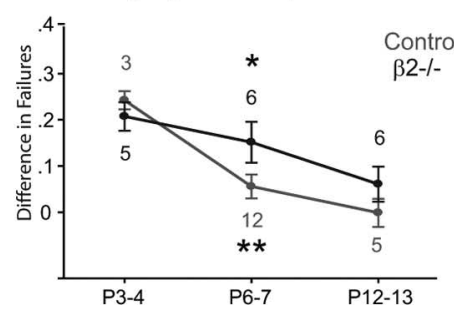

Figure 3. $\quad \beta 2^{-1-}$ animals retain a higher number of silent synapses than controls after the first postnatal week. $\boldsymbol{A}$, Example minimal stimulation experiment for a P7 control cell, showing amplitudes of all events (mixture of failures and successes) evoked at $-70 \mathrm{mV}$ holding potential and $+40 \mathrm{mV}$ holding potential. $\boldsymbol{B}$, Amplitude frequency histogram of example shown in $\boldsymbol{A}$, normalized to the number of events at each holding potential. Note similar peaks around $0 \mathrm{pA}$ for each curve ( $-70 \mathrm{mV}$ in black; $+40 \mathrm{mV}$ in gray). $\boldsymbol{C}$, Example traces from experiment shown in $\boldsymbol{A}$, showing AMPA-mediated successes and failures at $-70 \mathrm{mV}$ (left) and the mixed AMPA and NMDA currents at $+40 \mathrm{mV}$ (right). Stimulus artifacts have been removed for clarity. $D$, Example minimal stimulation experiment for a P7 $\beta 2^{-/-}$cell, as described in $\boldsymbol{A}$. $\boldsymbol{E}$, Example traces from experiment shown in $\boldsymbol{D}$, showing AMPA-mediated successes and failures at $-70 \mathrm{mV}$ (left) and the mixed AMPA and NMDA currents at $+40 \mathrm{mV}$ (right). Note appearance of purely NMDA-mediated responses at $+40 \mathrm{mV}$. $\boldsymbol{F}$, Normalized amplitude frequency histograms of example shown in $\boldsymbol{D}$. Note lower peak for $+40 \mathrm{mV}$ curve (gray) at $0 \mathrm{pA}$ relative to $-70 \mathrm{mV}$ curve (black). $\mathbf{G}$, Failure rates were computed for each experiment at each holding potential, and are depicted as paired ladder plots. Plots for all experiments in control cells (gray lines) and $\beta 2^{-/-}$cells (black lines) are shown at P3-P4 $\left(\boldsymbol{G}_{\mathbf{7}}\right), P 6-P 7\left(\boldsymbol{G}_{2}\right)$, and P12-P13 $\left(\boldsymbol{G}_{\mathbf{3}}\right) . \boldsymbol{H}$, Summary of the developmental profile for the prevalence of silent synapses in control and $\beta 2^{-1-}$ mice, as measured by the difference in failure rates for each experiment $(-70 \mathrm{mV}$ minus $+40 \mathrm{mV}$ ) averaged across groups. A lower failure rate at $+40 \mathrm{mV}$ is indicative of NMDAR-only synapses. A similarly high difference in failure rates is apparent at P3-P4 in both genotypes ( $p=0.5)$, revealing the presence of silent synapses early in retinocollicular development. In control synapses, there is a significant decrease in the mean difference in failure rates from P3-P4 to P6 - P7 (** $p<0.01)$, although it should be noted that some control cells still displayed lower failure rates at $+40 \mathrm{mV}\left(\mathbf{G}_{2}\right)$. This decrease fails to occur in $\beta 2^{-/-}$synapses, resulting in higher differences for $\beta 2^{-/-}$cells at P6 $-\mathrm{P7}$ relative to controls ( $\left.{ }^{*} p<0.05\right)$. The percentage of silent synapses returns to control levels by $\mathrm{P} 12-\mathrm{P} 13$ in $\beta 2^{-1-}$ cells ( $p=$ $0.25)$, suggesting a retinal-wave-dependent developmental program of synapse unsilencing in the retinocollicular pathway.
(Torborg et al., 2005). Furthermore, the information content of RGC activity during retinal waves relevant for refinement is contained on the order of seconds (Butts and Rokhsar, 2001). We therefore designed a "burst-based plasticity protocol" to mimic coincident RGC activity during retinal wave activity at retinocollicular synapses. After obtaining a stable baseline recording of AMPA-mediated current in voltage clamp, we switched the cell to current clamp and delivered a 1-s-long postsynaptic current injection to evoke spikes. The plasticity protocol consists of pairing this 1-s-long current injection with a coincident $20 \mathrm{~Hz}$ presynap- 
$\mathrm{A}_{1}$
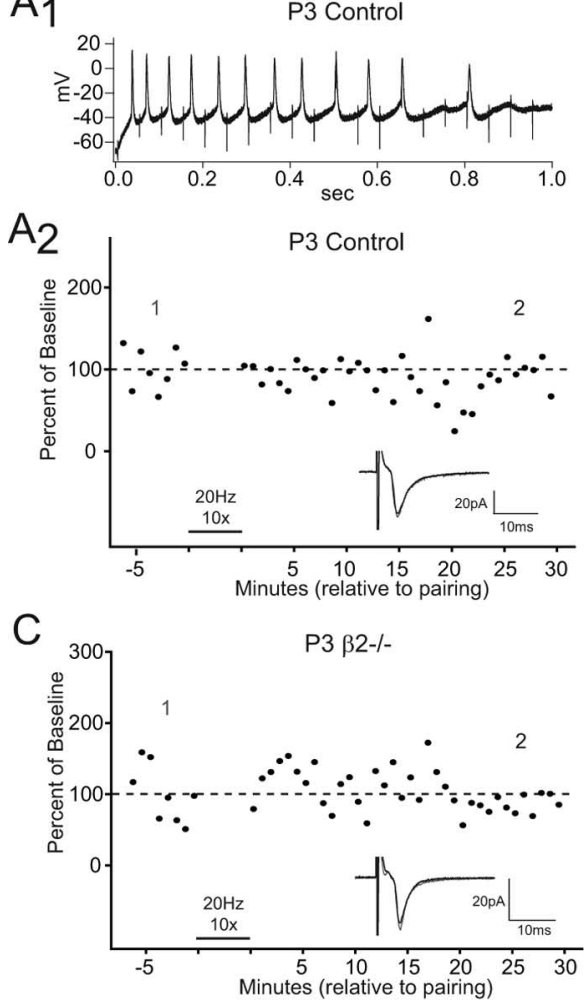

$\mathrm{B}_{1}$

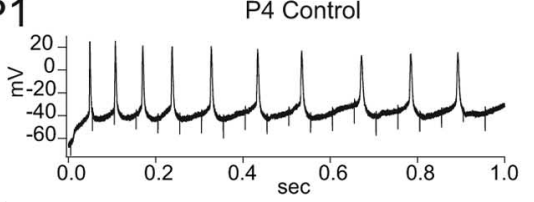

$\mathrm{B}_{2}$

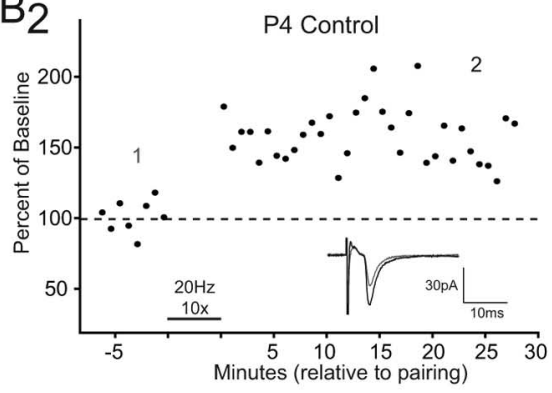

D

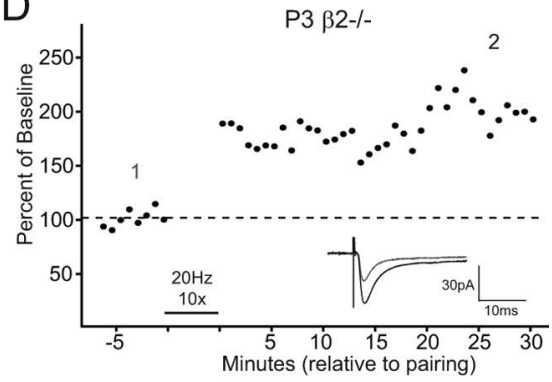

\section{E} Mean Change After Pairing

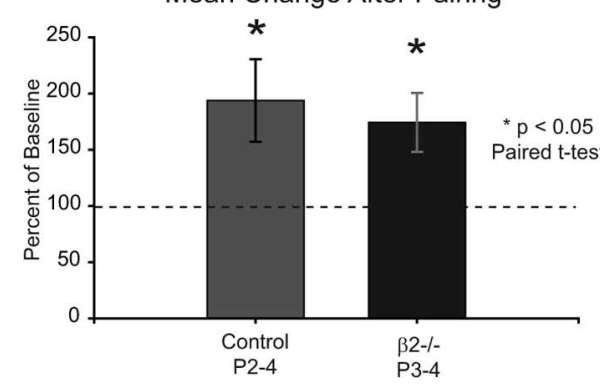

Figure 4. A coincident, burst-based pairing protocol results in heterogeneous plasticity, but LTP on average in control and $\beta 2^{-1-}$ synapses at P3-P4. To mimic coincident activity during retinal waves, our plasticity protocol consisted of pairing a 1-s-long postsynaptic current injection to the $\mathrm{SC}$ neuron under current clamp with a simultaneous 1-s-long $20 \mathrm{~Hz}$ presynaptic burst delivered extracellularly to RGC axons 10 times every 30 s, after which the recording was switched back to voltage clamp and the initial AMPA response amplitude monitored for any change. The burst-based plasticity protocol resulted in similar plasticity outcomes at P3-P4 in the control and $\beta 2^{-1-}$ populations that consisted of both LTP and no change in AMPA response amplitude, as shown in $\boldsymbol{A}_{2}, \boldsymbol{B}_{2}, \boldsymbol{C}$, and $\boldsymbol{D}$. All points in $\boldsymbol{A}_{2}-\boldsymbol{D}$ are the average of five sweeps and are normalized to the mean of the baseline. The dashed line represents $100 \%$ of baseline. The insets show example traces of the average of the last 5 min of the baseline $(1$, gray) and the average of the last 5 min of the recording $(2$, black), which were the values used to define plasticity during analysis. $A_{1}$, Example current-clamp record for one pairing during the plasticity protocol in a $\mathrm{P} 3$ control cell, depicting the spikes evoked postsynaptically and the $20 \mathrm{~Hz}$ stimulation artifacts. $A_{2}$, Pairing experiment in a P3 control cell (from $A_{7}$ ) that resulted in no change in AMPA response amplitude. $\boldsymbol{B}_{1}$, Example current-clamp record during pairing in a P4 control cell. $\boldsymbol{B}_{2}$, Experiment from a P4 control cell (from $\boldsymbol{B}_{7}$ ) that resulted in LTP. $\boldsymbol{C}$, Experiment in a P3 $\mathrm{B2}^{-/-}$cell that resulted in no change. $\boldsymbol{D}$, Experiment in a $\mathrm{P} 3 \mathrm{B2}^{-1-}$ cell that resulted in LTP. E, Summary of mean change after pairing. Each bar shows the mean change \pm SEM for all P2-P4 control cells (gray; $n=10$ ) and P3-P4 $\beta 2^{-1-}$ cells (black; $n=7$ ). Regardless of the heterogeneity in plasticity outcomes, LTP was induced on average in each group, as measured by a paired $t$ test $\left({ }^{*} p<0.05\right.$ ). These results suggest that the statistics of RGC bursting during retinal waves are capable of inducing synaptic strengthening at immature retinocollicular synapses, providing a potential mechanism for the observed synaptic maturation.

tic stimulation that also lasts for $1 \mathrm{~s}$, and repeating this pairing 10 times every $30 \mathrm{~s}$. Figure $4 A 1$ shows an example current-clamp record for a representative pairing in a $\mathrm{P} 3$ control cell, depicting the spikes evoked by postsynaptic current injection and the $20 \mathrm{~Hz}$ presynaptic stimulation artifacts. After the pairing, the cell is returned to voltage clamp, and the amplitude of the AMPA current is monitored for any change. A similar protocol was recently demonstrated to induce plasticity at the developing retinogeniculate synapse (Butts et al., 2007).

We first examined the effects of our plasticity protocol at $\mathrm{P} 3-\mathrm{P} 4$ (Fig. 4), an age at which all synaptic parameters are immature regardless of genotype. We find that our burst-based pairing protocol results in heterogeneous plasticity outcomes at P3-P4 in control (Fig. 4A2,B2) and $\beta 2^{-1-}$ (Fig. $4 C, D$ ) synapses. Figure $4, A 2$ and $C$, shows examples of experiments across genotypes resulting in no change in current amplitude, whereas Figure 4, B2 and D, depict experiments resulting in LTP. Regardless of this heterogeneity, however, we find that LTP is induced on average in both groups (Fig. 4E) (control P2-P4, $p<0.05$; $\beta 2^{-l-}$ P3-P4, $p<0.05$; paired $t$ test). These results suggest that in a population of synapses that are immature on average, the statistics of retinal waves are capable of inducing LTP on average under conditions of coincident presynaptic and postsynaptic bursting, supporting the hypothesis that retinocollicular synaptic strengthening and maturation during the first postnatal week occurs through a learning rule that is physiologically relevant to retinal waves.

We next examined the effects of the plasticity protocol at $\mathrm{P} 6-\mathrm{P} 7$, an age at which retinocollicular synapses have matured in control animals, but remain weak and immature in $\beta 2^{-1-}$ animals. We again find heterogeneous plasticity outcomes in both control (Fig. 5A2,B2) and $\beta 2^{-1-}$ cells (Fig. $5 C, D)$. However, at this age, many fewer control cells showed LTP (summary in Fig. $6 C$ ), resulting in no change on average (Fig. $5 E)(p=0.5$, paired $t$ test). If the LTP observed at P3-P4 is a property of an immature synaptic network with a high capacity for LTP induction, then we would predict that the immature $\beta 2^{-/-}$synaptic network at P6-P7 would also show LTP on average in the face of appropriate coincident activity. This increase is indeed what we observe in the $\beta 2^{-1-}$ group at P6-P7 (Fig. 5E) $(p<0.05$, paired $t$ test $)$. These results suggest that, after synaptic strengthening has already occurred, the capacity for additional LTP is greatly diminished, and this may reflect the closure of a "critical period" for both synaptic plasticity and retinocollicular map refinement. If synaptic strengthening is delayed, however, this developmental window for LTP induction is extended.

A summary of all plasticity outcomes across ages and genotypes is shown in Figure 6, plotted as frequency histograms and placed into $10 \%$ bins. We first examined whether the distributions differed as a function of age and genotype, and found that the plasticity outcomes in the control P3-P4 group are different 
from the control P6-P7 group (control P3-P4 vs control P6-P7, $p<0.05$; MannWhitney $U$ test). The distributions in the $\beta 2^{-1-} \mathrm{P} 3-\mathrm{P} 4$ group and the $\beta 2^{-1-} \mathrm{P} 6-\mathrm{P} 7$ group, however, are not different, suggesting the capacity for plasticity remains immature in the mutants $\left(\beta 2^{-/-} \mathrm{P} 3-\mathrm{P} 4\right.$ vs $\beta 2^{-1-}$ P6-P7, $p=0.6$; Mann-Whitney $U$ test). Accordingly, the P6-P7 $\beta 2^{-1-}$ group is also different from the $\mathrm{P} 6-\mathrm{P} 7$ control group $\left(\beta 2^{-1-} \mathrm{P} 6-\mathrm{P} 7\right.$ vs control P6-P7, $p<0.05$; Mann-Whitney $U$ test). We also found that the probability of inducing LTP is different in the immature groups relative to the mature control group. For this analysis, we defined LTP as $>120 \%$ of baseline, and compared the fraction of cells that showed LTP across groups. In control cells, the frequency of inducing LTP is greater at P3-P4 than at P6-P7 (control P3-P4, 7 of 10; control P6-P7, 2 of $12 ; p<0.05, \chi^{2}$ test). Among $\beta 2^{-1-}$ cells, however, the frequency of inducing LTP is similar at both ages $\left(\beta 2^{-1-} \mathrm{P} 3-\mathrm{P} 4,4\right.$ of $7 ; \beta 2^{-/-} \mathrm{P} 6-\mathrm{P} 7,9$ of $16 ; p=0.9, \chi^{2}$ test). Furthermore, the proportion of LTP is significantly greater in $\beta 2^{-1-} \mathrm{P} 6-\mathrm{P} 7$ cells than in control P6-P7 cells ( $p<0.05, \chi^{2}$ test), confirming that the capacity for LTP differs among the immature and mature populations. Combined with the paired $t$ test results from Figures 4 and 5, we conclude that a burst-based learning rule can be used to induce LTP in weak, immature synapses regardless of age or genotype. After synaptic strengthening and maturation have occurred, the probability of inducing LTP is greatly diminished, although not completely lost.

We also found that the burst-based plasticity protocol often led to a reduction in the number of AMPA-mediated failures. An experimentally induced reduction in failure rates is typically attributed to an "unsilencing" of NMDAR-only synapses through the trafficking of AMPARs into the synapse (Liao et al., 1995; Durand et al., 1996; Isaac et al., 1997). In the subset of cells that had an initially high failure rate during the baseline $(>30 \%)$, the plasticity protocol always induced a reduction in the number of failures, usually accompanied by a large increase in the amplitude of the successes (Fig. 7). Interestingly, a large reduction of failures was also observed in the P6 control cell with the largest potentiation (Figs. 6C, 7D). As described above, the prevalence of silent synapses decreases over the first postnatal week in control animals, but some are maintained (Fig. 3G). These results suggest that the plasticity is not dependent on the genotype, but on the state of maturity of the synapse; in the small subset of silent, immature control synapses at P6-P7, the plasticexplanation).
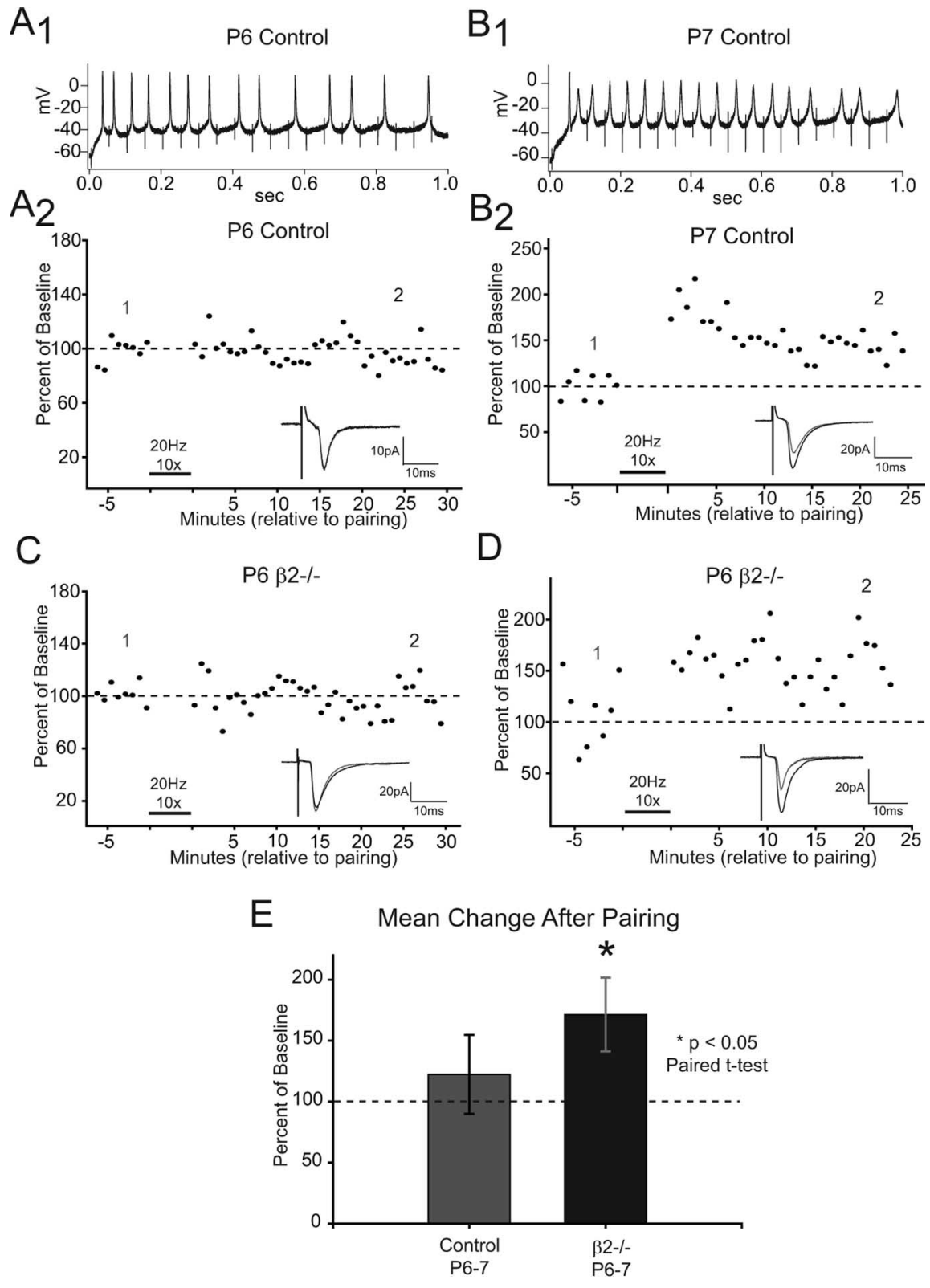

Figure 5. The capacity for LTP is reduced by $P 6-P 7$ in more mature control synapses, but is retained among immature $\beta 2^{-1-}$ synapses. The plasticity protocol used at P6 - P7 was the same as described in Figure 4 for P3-P4 cells, and all graphs in $\boldsymbol{A}_{2}-D$ are plotted as described in Figure $4, \boldsymbol{A}_{2}-\boldsymbol{D}$. $\boldsymbol{A}_{1}$, Example current-clamp record during a pairing for a $\mathrm{P} 6$ control cell, depicting the spikes evoked postsynaptically and the $20 \mathrm{~Hz}$ stimulation artifacts. $\boldsymbol{A}_{2}$, Experiment from a P6 control cell (from $\boldsymbol{A}_{7}$ ) that resulted in no change in AMPA response amplitude. $\boldsymbol{B}_{1}$, Example current-clamp record during a pairing for a $P 7$ control cell. $\boldsymbol{B}_{2}$, Experiment from a P7 control cell (from $\boldsymbol{B}_{1}$ ) that resulted in LTP. C, Experiment from a P6 $\beta 2^{-1-}$ cell that resulted in no change. $D$, Experiment from a P6 $\beta 2^{-1-}$ cell that resulted in LTP. E, Summary of mean change after pairing, where plasticity was defined as the average amplitude of the last $5 \mathrm{~min}$ of the recording compared with the average amplitude of the last $5 \mathrm{~min}$ of the baseline. Each bar shows the mean change \pm SEM after pairing for all control cells (gray; $n=12$ ) and $\beta 2^{-1-}$ cells (black; $\left.n=16\right)$ at P6 -P7. At this age, many fewer control cells displayed LTP, resulting in no change average (paired $t$ test, $p=0.5$ ). The immature $\beta 2^{-1-}$ synapses, however, still retained the ability to show LTP on average, as measured using a paired $t$ test $\left({ }^{*} p<0.05\right)$. This result reveals that synaptic populations that are immature on average also show LTP on average, providing a potential mechanism for the observed synaptic maturation. After synaptic strengthening and maturation has already occurred, the ability to undergo LTP is greatly diminished, perhaps reflecting a metaplastic model of circuit development (see Discussion for

ity protocol is still able to induce a robust LTP characteristic of all other immature synapses at P3-P4 and $\beta 2^{-/-}$synapses at P6-P7.

Burst-based synaptic plasticity depends on coincident activity To examine the effects of presynaptic versus postsynaptic stimulation alone and to verify the Hebbian nature of the plasticity, we 
A

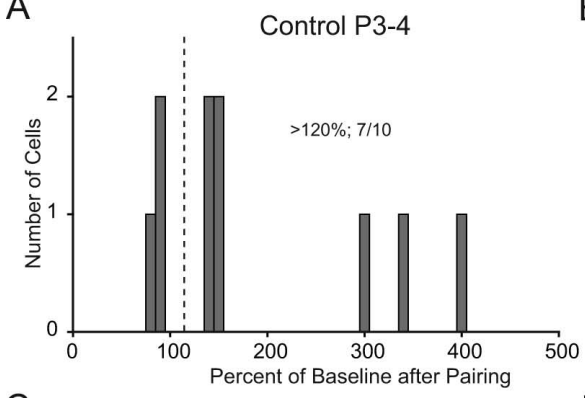

C

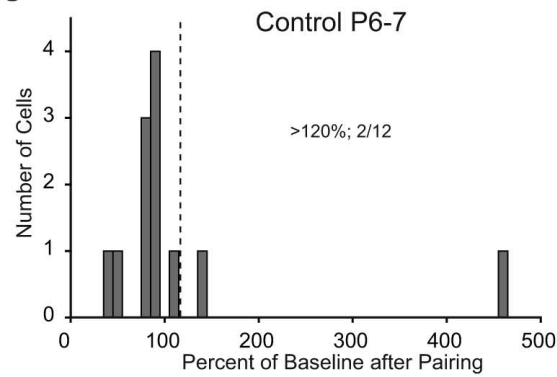

$\mathrm{B}$

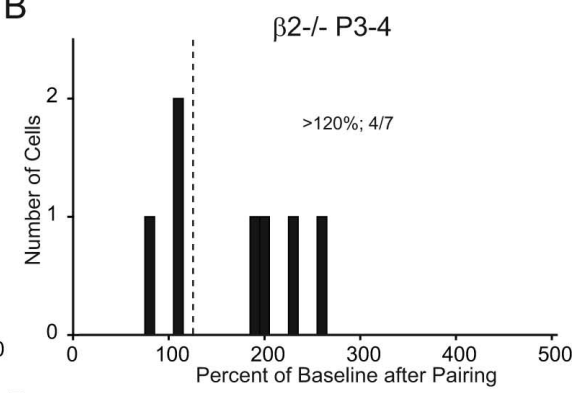

D

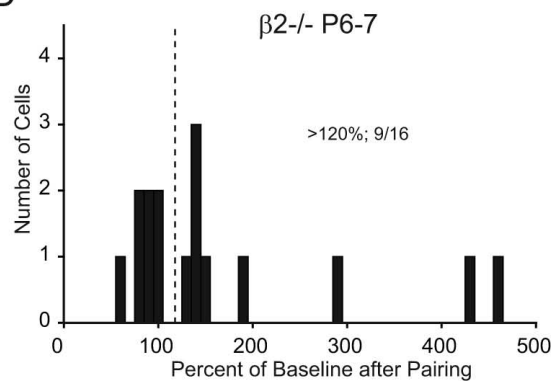

Figure 6. Summary of all plasticity outcomes at P3-P4 and P6 -P7. All plasticity outcomes are expressed as percentage of baseline, placed into $10 \%$ bins, and plotted as frequency histograms. LTP was defined as $>120 \%$ of baseline, and no change was defined as $80-120 \%$ of baseline. The vertical dashed line represents $120 \%$ threshold. $A$, Summary of all control P3-P4 experiments, with 7 of 10 showing LTP. $\boldsymbol{B}$, Summary of all $\beta 2^{-1-}$ P3-P4 experiments, with 4 of 7 showing LTP. $\boldsymbol{C}$, Summary of all control P6 -P7 experiments, with 2 of 12 showing LTP. D, Summary of all $\beta 2^{-1-} P 6-P 7$ experiments, with 9 of 16 showing LTP. Using a Mann-Whitney $U$ test, we find that the control P3-P4 distribution is different from the control P6 $-P 7$ distribution ( $p<$ 0.05 ), whereas the $\beta 2^{-1-}$ distributions at P3-P4 and P6 $-\mathrm{P} 7$ are not significantly different $(p=0.6)$. The $\beta 2^{-1-} \mathrm{P} 6-\mathrm{P} 7$ distribution is, however, different from the control P6 $-P 7$ group $(p<0.05)$. Using $\chi^{2}$ statistics, we also tested whether the frequency of LTP differs across ages and genotypes. The probability of finding LTP is greater in control P3-P4 cells than in control $P 6-P 7$ cells (control P3-P4, 7 of 10 ; control P6 - P7, 2 of 12; $p<0.05$ ), whereas the frequency of LTP remains similar across ages for $\beta 2^{-1-}$ cells $\left(\beta 2^{-1-} P 3-P 4,4\right.$ of $7 ; \beta 2^{-1-} P 6-P 7,9$ of $\left.16 ; p=0.9\right)$. Accordingly, the frequency of LTP is greater in $\beta 2^{-1-} \mathrm{P} 6-\mathrm{P} 7$ cells than in control P6 -P7 cells (control P6 $-\mathrm{P7}, 2$ of 12; $\beta 2^{-1-} \mathrm{P} 6-\mathrm{P7}, 9$ of $16 ; p<0.05$ ). These results confirm that the plasticity outcomes differ among the immature and mature synaptic populations, and that the probability of inducing LTP decreases after synaptic strengthening and maturation have occurred.

repeated the plasticity experiments at $\mathrm{P} 6-\mathrm{P} 7$, but separated the onset of the 1-s-long $20 \mathrm{~Hz}$ presynaptic burst and the 1-s-long postsynaptic current injection by $3 \mathrm{~s}$. This protocol maintains similar presynaptic and postsynaptic activity levels, but eliminates the coincident activity between RGCs and target SC neurons expected during retinal wave activity. Figure $8, A 1$ and $B 1$, shows example current-clamp records during these noncoincident pairings. Figure $8, A 2$ and $B 2$, depicts the result of these plasticity experiments from a P7 control and P7 $\beta 2^{-1-}$ cell, each resulting in no change. The summary histograms are displayed in Figure 8, $C$ and $D$, constructed as described in Figure 6. Under these noncoincident conditions, LTP was never observed (control P6-P7, $p=0.6 ; \beta 2^{-1-} \mathrm{P} 6-\mathrm{P} 7, p=0.5$, paired $t$ test), and the $\beta 2^{-I-}$ distribution was not statistically different from the control distribution (Mann-Whitney $U$ test, $p=0.8$ ). We conclude that the burst-based learning rule depends on coincident activity, and that presynaptic or postsynaptic stimulation alone is not sufficient to induce LTP at retinocollicular synapses, supporting the hypothesis that correlated activity is a necessary substrate for synaptic strengthening during the period of retinotopic map refinement.

\section{Discussion}

\section{Summary}

Previous studies of the $\beta 2^{-/-}$mouse showed that correlated retinal waves during the first postnatal week are necessary for retinotopic map refinement in the superior colliculus (McLaughlin et al., 2003; Chandrasekaran et al., 2005). In this study, we find an increase in AMPA/ NMDA ratios at retinocollicular synapses during the first postnatal week that is attributable to both an increase in the synaptic AMPA component and a decrease in the number of silent synapses. In the absence of correlated retinal waves, these functional changes are prevented in $\beta 2^{-1-}$ mice at $\mathrm{P} 6-\mathrm{P} 7$. These parameters return to control levels by P12-P13 in $\beta 2^{-/-}$animals, suggesting that the delayed onset of correlated retinal waves is able to drive synapse maturation. Using a seconds-long "burst-based" plasticity protocol designed to mimic the statistics of RGC bursting during natural retinal waves, we find that LTP is induced on average across all immature populations. The capacity for LTP is greatly diminished in the more mature control population at $\mathrm{P} 6-\mathrm{P} 7$, a period near the end of retinotopic map refinement.

\section{Model of retinal-wave-dependent synaptic plasticity}

Our findings suggest that retinocollicular synaptic maturation is dependent on correlated activity and can occur through a learning rule that is physiologically relevant to retinal waves. Retinal wave activity consists of correlated bursts of action potentials that sweep across the retina, providing statistics appropriate for Hebbianbased synaptic refinement (Butts, 2002). Analysis of anatomical patterning in the LGN reveals that the relevant statistics for topographic instruction are highfrequency bursting and correlated firing among neighboring RGCs (Torborg et al., 2005), and information theory shows that the relevant timescale for a synaptic learning rule based on retinal wave activity is on the order of seconds (Butts and Rokhsar, 2001). In the context of retinotopic map refinement, therefore, a seconds-long burst-based learning rule would make more effective use of retinal waves than a classical spike-timing-dependent learning rule, which acts on the order of milliseconds (Dan and Poo, 2004). It should be noted that other wave statistics, such as RGC firing rate during bursts, could also influence LTP induction. A characterization of the dependence of burst-based LTP on firing rate would be worthy of additional investigation.

A recent study at the developing retinogeniculate synapse demonstrated that a burst-timing-dependent plasticity rule can lead to synaptic enhancement and, interestingly, that the underlying spike timings on the millisecond timescale cannot fully account for the observed plasticity (Butts et al., 2007). These results were seen during a period of significant refinement and maturation of retinogeniculate synapses, including increases in AMPA/ NMDA ratios and decreases in the presence of silent synapses (Chen and Regehr, 2000). By varying the timings of presynaptic and postsynaptic burst onsets, Butts et al. (2007) also demonstrated that LTP did not depend on burst order, and that longterm depression (LTD) could be induced by separating burst onsets by hundreds to thousands of milliseconds. This supports a model in which the refinement of retinofugal projections not 
only involves the strengthening of appropriate synapses via an LTP-like mechanism but also requires the weakening and elimination of inappropriate synapses through LTD-like mechanisms. Although we have not examined the windows for LTD induction at the retinocollicular synapse, we find that a 3-s-long separation between burst onsets is not capable of inducing plasticity (Fig. 8). Combined, these data are consistent with a "symmetric" learning rule that regulates synaptic maturation in the SC and LGN by reading out coincident bursts among RGCs during retinal wavemediated map refinement.

\section{Model of synaptic-competition-based retinotopic map refinement}

Whether such synaptic maturation and plasticity is required for map refinement, however, remains unknown, but evidence supporting such a model comes from numerous studies. This form of activity-dependent competition is thought to be mediated by NMDARs, because their dual ligand and voltage dependence allows for detection of coincident presynaptic and postsynaptic activity (Constantine-Paton and Cline, 1998; Zhang and Poo, 2001). In the frog retinotectal system, an NMDAR-dependent LTP-like process results in a developmental increase in AMPA/NMDA ratios at retinotectal synapses during map refinement (Wu et al., 1996). Interestingly, chronic NMDAR blockade serves to increase RGC arbor branch dynamics, suggesting that NMDAR activity and synaptic maturation normally stabilizes retinotectal projections during circuit formation (Rajan et al., 1999). Recent in vivo imaging of zebrafish and tadpole retinotectal axons reveals that synapse formation and maturation are tightly correlated with branch dynamics and may play an instructive role in arbor remodeling during map formation (Meyer and Smith, 2006; Ruthazer et al., 2006). Indeed, correlated activity is known to regulate RGC axon branch dynamics during development (Ruthazer et al., 2003; Hua et al., 2005), offering additional evidence that the refinement of retinotopic projections proceeds through activitydependent instruction and synaptic competition.

In the rodent superior colliculus, chronic NMDAR blockade disrupts retinocollicular topography (Simon et al., 1992) and increases the sprouting of RGC axons and the density of retinocollicular synapses during the period of map refinement (Colonnese and Constantine-Paton, 2006). This supports the hypothesis that synaptic competition stabilizes developing axons and eliminates inappropriate connections in mammals as well. We previously showed that $\beta 2^{-1-}$ synapses at P6-P7 are not only weaker but also greater in number relative to control synapses (Chandrasekaran et al., 2007). Correlated presynaptic activity, therefore, may be the necessary upstream signal for synaptic learning rules to strengthen appropriate synapses and eliminate inappropriate inputs.

\section{Role of unsilencing of synapses during activity-dependent development}

Another model for neural circuit development involves the initial formation of silent "candidate" synapses during axons out-
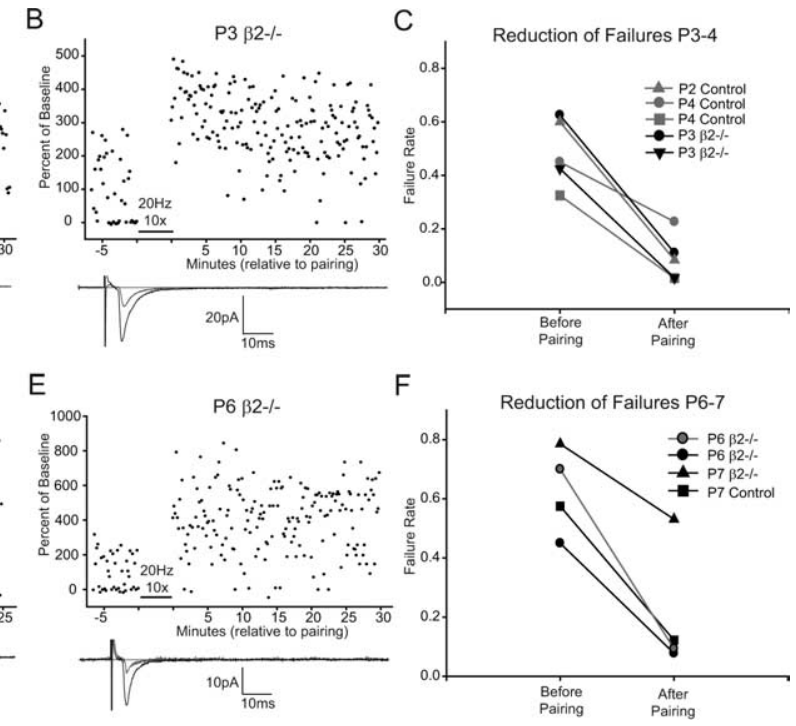

Figure 7. The burst-based plasticity protocol is able to unsilence synapses across all ages and genotypes. In the subset of reduction of failure experiments at P2-P4, with the failure rate before and after pairing shown for three control cells and two noted that a true unsilencing is not confirmed, because we were not able to compare the failure rates at $-70 \mathrm{mV}$ against those at $+40 \mathrm{mV}$ before and after pairing for each experiment.

growth, and their conversion to functional inputs requires coincident activity that represents salient information (Feldman et al., 1999). As relevant connections are made functional, therefore, there should be a concurrent developmental downregulation of silent synapses, and indeed such a profile has been observed in numerous brain regions (Isaac et al., 1995, 1997; Wu et al., 1996; Rumpel et al., 1998; Franks and Isaacson, 2005). Additionally, this disappearance of silent synapses in many circuits coincides with the closure of the critical period for LTP induction (Crair and Malenka, 1995; Kirkwood et al., 1995), suggesting a mechanistic link. In direct support of this, experimental induction of LTP at putative silent synapses has been shown to convert NMDAR-only synapses into AMPAR-containing synapses, thereby rendering them functional at resting membrane potential (Liao et al., 1995; Durand et al., 1996; Wu et al., 1996; Isaac et al., 1997). Our results support such a model during the refinement of retinocollicular synapses, because correlated activity produces a developmental downregulation of silent synapses. Furthermore, we provide evidence that a burst-based learning rule can lead to LTP via the unsilencing of immature synapses (Fig. 7), providing a mechanism for circuit formation.

\section{Source of heterogeneous plasticity and metaplastic model of circuit development}

Although we find that the burst-based learning rule leads to LTP on average across immature synaptic populations, we observe a heterogeneous plasticity outcome. Similar results at multiple synapses have also been observed using a variety of timingdependent learning rules (Debanne et al., 1999; Montgomery et al., 2001; Hardingham et al., 2007), and a number of explanations for this heterogeneity have been proposed. Timing-dependent 

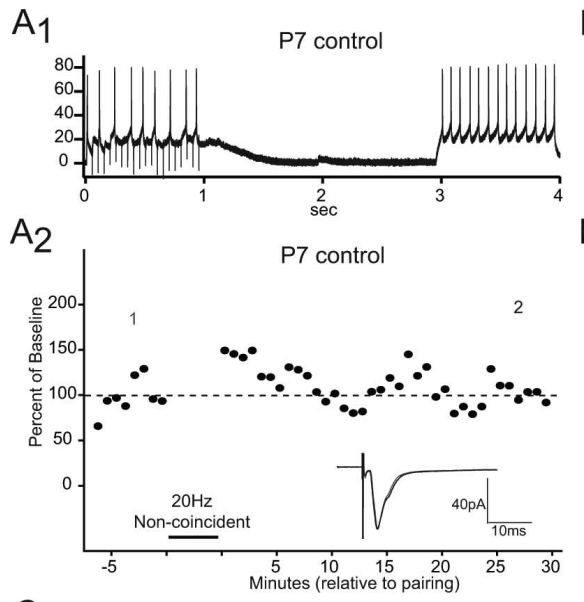

C
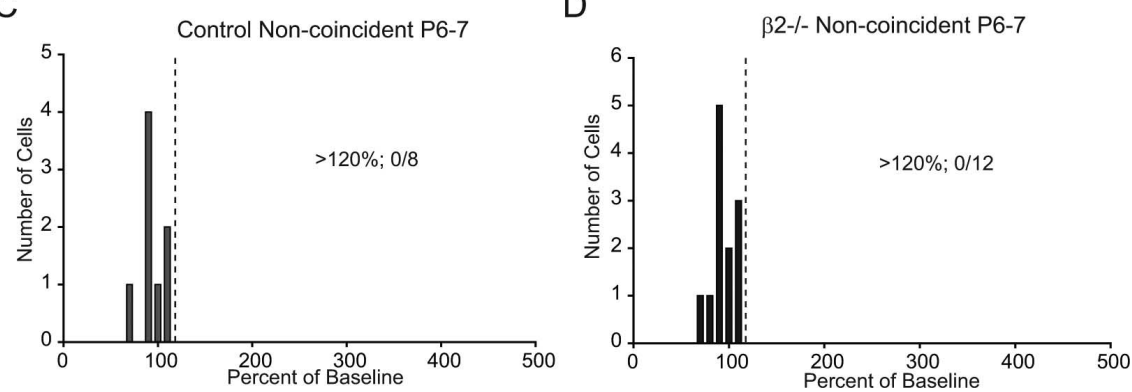

Figure 8. The burst-based learning rule is Hebbian and requires coincident presynaptic and postsynaptic activity. To test the effects of presynaptic and postsynaptic stimulation alone on retinocollicular synaptic efficacy, we applied the same burst-based plasticity protocol at $\mathrm{P} 6-\mathrm{P} 7$, but separated the onset of the presynaptic burst and the postsynaptic current injection by $3 \mathrm{~s}$. Here, we are eliminating the coincident activity between RGCS and SC neurons, a necessary substrate for Hebbian changes. All plasticity experiments are plotted as described in Figure $4, A_{2}-D$. $A_{1}$, Example current-clamp record from a noncoincident pairing experiment for a P7 control cell. The first second is the $20 \mathrm{~Hz}$ presynaptic stimulation alone, and the last second is the postsynaptic current injection. $\boldsymbol{A}_{2}$, Experiment for a P7 control cell (from $\boldsymbol{A}_{1}$ ) that resulted in no change in AMPA response amplitude. $\boldsymbol{B}_{1}$, Example current-clamp record from a noncoincident pairing experiment for a P6 $\beta 2^{-1-}$ cell, as described in $\boldsymbol{A}_{1}$. $\boldsymbol{B}_{2}$, Experiment for a P6 $\beta 2^{-/-}$cell (from $\boldsymbol{B}_{1}$ ) that resulted in no change in AMPA response. $\boldsymbol{C}$, Summary of all noncoincident plasticity experiments at P6-P7 for control cells. All plasticity outcomes are expressed as percentage of baseline, placed into $10 \%$ bins, and plotted as frequency histograms. D, Summary of all noncoincident plasticity experiments at P6 $-\mathrm{P7}$ for $\beta 2^{-1-}$ cells, as described for $C$. Under these noncoincident conditions, LTP was never induced in either groups ( 0 of 8 for controls; 0 of 12 for $\beta 2^{-l-}$ ). The paired $t$ test reveals no change on average (control $\mathrm{P} 6-\mathrm{P} 7, p=0.6 ; \beta 2^{-1-} \mathrm{P} 6-\mathrm{P} 7, p=0.5$ ), and the Mann-Whitney $U$ test reveals that the control and $\beta 2^{-1-}$ distributions are not significantly different from one another $(p=0.8)$. These results confirm that coincident bursting activity is required to induce plasticity at retinocollicular synapses, consistent with a Hebbian synaptic learning rule.

learning rules have been shown to depend on dendritic synapse location in various brain areas, with both LTP and LTD being observed under the same induction protocol but applied to synapses at various distances from the soma (Froemke et al., 2005; Sjostrom and Hausser, 2006). Additionally, the plasticity outcome of the same synaptic learning rule can depend on which cell type is being studied (Bi and Poo, 1998; Tzounopoulos et al., 2004). These factors were not controlled for in this study and are worthy of additional investigation given the heterogeneity in cell types and dendritic morphology present in the superficial SC (Endo and Isa, 2001).

Heterogeneous plasticity has also been linked to models of metaplasticty, in which the current state of a synapse can regulate its capacity for future plasticity. In the hippocampus and visual cortex, for example, the sign and magnitude of plasticity correlates with initial synaptic strength, with weaker synapses showing LTP and stronger synapses showing LTD (Bi and Poo, 1998; Montgomery et al., 2001; Hardingham et al., 2007). Accordingly, neural activity can dynamically gate the threshold for plasticity by regulating synaptic maturation. For example, sensory depriva- tion experiments that result in a delayed maturation of cortical synapses also result in a greater potential for LTP at these immature synapses (Kirkwood et al., 1995, 1996; Franks and Isaacson, 2005). The effects of eliminating cholinergic waves in $\beta 2^{-1-}$ mice appears to be analogous to the effects of sensory deprivation, because synaptic maturation and plasticity at target structures are delayed, but not prevented. Although we cannot make synapse-bysynapse correlations with initial synaptic strength and LTP based on the nature of our extracellular stimulation, we find that synaptic populations that are immature and weak on average also show LTP on average, whereas the more mature control population at P6-P7 does not. Furthermore, the small percentage of synapses that are putatively silent and weak even in this mature control population also show a robust LTP under the burst-based learning rule, supporting a metaplastic model for retinocollicular map refinement.

\section{Comparison with previous work on synaptic maturation and plasticity in the rodent $\mathrm{SC}$}

Activity-dependent synaptic maturation has been previously documented in the superficial layer of the SC, but at later ages than those reported here. Chronically disrupting NMDAR activity prevents the normal developmental increase in AMPA/ NMDA ratios seen after the second postnatal week (Shi et al., 2001; Colonnese et al., 2003). Additionally, the onset of patterned visual experience leads to a number of synaptic changes after eye opening similar to those observed in this study over the first postnatal week. A transient increase in silent synapses immediately after eye opening is subsequently decreased by the addition of AMPAR to both new and existing synapses ( $\mathrm{Lu}$ and Constantine-Paton, 2004). Because the projections from the visual cortex to the SC are refining during this period, these results suggest that nascent corticocollicular synapses may undergo similar activity-dependent changes as developing retinocollicular synapses during topographic refinement.

NMDAR-dependent LTP has also been observed in the SC after eye opening and was proposed as the mechanism for the synaptic changes described above ( $\mathrm{Lu}$ and Constantine-Paton, 2004; Zhao et al., 2006). Interestingly, the $20 \mathrm{~Hz}$ protocol used in that study failed to induce plasticity before eye opening (Zhao and Constantine-Paton, 2004). This could reflect the capacity for immature and silent corticocollicular synapses to undergo LTP, whereas the mature retinocollicular synapses immediately before eye opening have a much lower capacity for LTP, as shown in our study. Numerous other studies have also documented LTP during the first and second postnatal week in the SC (Hirai and Okada, 1993; Miyamoto and Okada, 1993; Lo and Mize, 2002), but these studies used field potential analysis, brainstem preparations, and various forms of high-frequency stimulation, which 
make it difficult to understand the cellular changes that occur during plasticity and their relevance to retinal-wave-dependent synaptic maturation.

\section{Conclusion}

The mechanisms of LTP have been widely hypothesized to be essential for the maturation and refinement of synapses during topographic map formation (Constantine-Paton and Cline, 1998). Numerous studies have revealed developmentally regulated synaptic maturation during periods when the expression of LTP is also robust (Crair and Malenka, 1995; Rumpel et al., 1998; Chen and Regehr, 2000), and this synaptic maturation is correlated with axon remodeling during map refinement (Meyer and Smith, 2006; Ruthazer et al., 2006). Whether the experimentally induced LTP is physiologically relevant to circuit formation is unclear, however, because of the wide variety of induction protocols used and the lack of information about natural activity patterns during development. The mouse retinocollicular map provides a unique model system to address this question, because the statistics of retinal waves and anatomical map refinement have been well documented over the first 2 postnatal weeks (McLaughlin et al., 2003; Chandrasekaran et al., 2005). We find that the period of retinotopic map refinement involves specific synaptic changes, including an increase in AMPAR currents and a decrease in the presence of silent synapses. We also find that mimicking retinal wave activity is sufficient to induce LTP on average among immature synapses, suggesting that a burst-based learning rule exists at retinocollicular synapses and may be used to read out retinal wave information to guide the strengthening of topographically appropriate connections. Our results support a model in which the activity-dependent instruction of map refinement occurs through the unsilencing and strengthening of an immature synaptic network through a Hebbian learning rule that is physiologically relevant to the statistics of its inputs.

\section{References}

Bansal A, Singer JH, Hwang BJ, Xu W, Beaudet A, Feller MB (2000) Mice lacking specific nicotinic acetylcholine receptor subunits exhibit dramatically altered spontaneous activity patterns and reveal a limited role for retinal waves in forming $\mathrm{ON}$ and OFF circuits in the inner retina. J Neurosci 20:7672-7681.

Bi GQ, Poo MM (1998) Synaptic modifications in cultured hippocampal neurons: dependence on spike timing, synaptic strength, and postsynaptic cell type. J Neurosci 18:10464-10472.

Butts DA (2002) Retinal waves: implications for synaptic learning rules during development. Neuroscientist 8:243-253.

Butts DA, Rokhsar DS (2001) The information content of spontaneous retinal waves. J Neurosci 21:961-973.

Butts DA, Kanold PO, Shatz CJ (2007) A burst-based "Hebbian" learning rule at retinogeniculate synapses links retinal waves to activity-dependent refinement. PLoS Biol 5:e61.

Chandrasekaran AR, Plas DT, Gonzalez E, Crair MC (2005) Evidence for an instructive role of retinal activity in retinotopic map refinement in the superior colliculus of the mouse. J Neurosci 25:6929-6938.

Chandrasekaran AR, Shah RD, Crair MC (2007) Developmental homeostasis of mouse retinocollicular synapses. J Neurosci 27:1746-1755.

Chen C, Regehr WG (2000) Developmental remodeling of the retinogeniculate synapse. Neuron 28:955-966.

Colonnese MT, Constantine-Paton M (2006) Developmental period for $\mathrm{N}$-methyl-D-aspartate (NMDA) receptor-dependent synapse elimination correlated with visuotopic map refinement. J Comp Neurol 494:738-751.

Colonnese MT, Shi J, Constantine-Paton M (2003) Chronic NMDA receptor blockade from birth delays the maturation of NMDA currents, but does not affect AMPA/kainate currents. J Neurophysiol 89:57-68.

Constantine-Paton M, Cline HT (1998) LTP and activity-dependent synaptogenesis: the more alike they are, the more different they become. Curr Opin Neurobiol 8:139-148.
Crair MC (1999) Neuronal activity during development: permissive or instructive? Curr Opin Neurobiol 9:88-93.

Crair MC, Malenka RC (1995) A critical period for long-term potentiation at thalamocortical synapses. Nature 375:325-328.

Dan Y, Poo MM (2004) Spike timing-dependent plasticity of neural circuits. Neuron 44:23-30.

Debanne D, Gahwiler BH, Thompson SM (1999) Heterogeneity of synaptic plasticity at unitary CA3-CA1 and CA3-CA3 connections in rat hippocampal slice cultures. J Neurosci 19:10664-10671.

Durand GM, Kovalchuk Y, Konnerth A (1996) Long-term potentiation and functional synapse induction in developing hippocampus. Nature 381:71-75.

Endo T, Isa T (2001) Functionally different AMPA-type glutamate receptors in morphologically identified neurons in rat superficial superior colliculus. Neuroscience 108:129-141.

Feldman DE, Nicoll RA, Malenka RC (1999) Synaptic plasticity at thalamocortical synapses in developing rat somatosensory cortex: LTP, LTD, and silent synapses. J Neurobiol 41:92-101.

Franks KM, Isaacson JS (2005) Synapse-specific downregulation of NMDA receptors by early experience: a critical period for plasticity of sensory input to olfactory cortex. Neuron 47:101-114.

Froemke RC, Poo MM, Dan Y (2005) Spike-timing-dependent synaptic plasticity depends on dendritic location. Nature 434:221-225.

Hardingham NR, Hardingham GE, Fox KD, Jack JJ (2007) Presynaptic efficacy directs normalization of synaptic strength in layer $2 / 3$ rat neocortex after paired activity. J Neurophysiol 97:2965-2975.

Hirai H, Okada Y (1993) Ipsilateral corticotectal pathway inhibits the formation of long-term potentiation (LTP) in the rat superior colliculus through GABAergic mechanism. Brain Res 629:23-30.

Hua JY, Smear MC, Baier H, Smith SJ (2005) Regulation of axon growth in vivo by activity-based competition. Nature 434:1022-1026.

Isaac JT, Nicoll RA, Malenka RC (1995) Evidence for silent synapses: implications for the expression of LTP. Neuron 15:427-434.

Isaac JT, Crair MC, Nicoll RA, Malenka RC (1997) Silent synapses during development of thalamocortical inputs. Neuron 18:269-280.

Katz LC, Shatz CJ (1996) Synaptic activity and the construction of cortical circuits. Science 274:1133-1138.

Kirkwood A, Lee HK, Bear MF (1995) Co-regulation of long-term potentiation and experience-dependent synaptic plasticity in visual cortex by age and experience. Nature 375:328-331.

Kirkwood A, Rioult MC, Bear MF (1996) Experience-dependent modification of synaptic plasticity in visual cortex. Nature 381:526-528.

Liao D, Hessler NA, Malinow R (1995) Activation of postsynaptically silent synapses during pairing-induced LTP in CA1 region of hippocampal slice. Nature 375:400-404.

Lo FS, Mize RR (2002) Properties of LTD and LTP of retinocollicular synaptic transmission in the developing rat superior colliculus. Eur J Neurosci 15:1421-1432.

Lu HC, She WC, Plas DT, Neumann PE, Janz R, Crair MC (2003) Adenylyl cyclase I regulates AMPA receptor trafficking during mouse cortical "barrel" map development. Nat Neurosci 6:939-947.

Lu W, Constantine-Paton M (2004) Eye opening rapidly induces synaptic potentiation and refinement. Neuron 43:237-249.

Malinow R, Malenka RC (2002) AMPA receptor trafficking and synaptic plasticity. Annu Rev Neurosci 25:103-126.

McLaughlin T, Torborg CL, Feller MB, O'Leary DD (2003) Retinotopic map refinement requires spontaneous retinal waves during a brief critical period of development. Neuron 40:1147-1160.

Meyer MP, Smith SJ (2006) Evidence from in vivo imaging that synaptogenesis guides the growth and branching of axonal arbors by two distinct mechanisms. J Neurosci 26:3604-3614.

Miyamoto T, Okada Y (1993) NMDA receptor, protein kinase C and calmodulin system participate in the long-term potentiation in guinea pig superior colliculus slices. Brain Res 605:287-292.

Montgomery JM, Pavlidis P, Madison DV (2001) Pair recordings reveal allsilent synaptic connections and the postsynaptic expression of long-term potentiation. Neuron 29:691-701.

Muir-Robinson G, Hwang BJ, Feller MB (2002) Retinogeniculate axons undergo eye-specific segregation in the absence of eye-specific layers. J Neurosci 22:5259-5264.

Rajan I, Witte S, Cline HT (1999) NMDA receptor activity stabilizes presyn- 
aptic retinotectal axons and postsynaptic optic tectal cell dendrites in vivo. J Neurobiol 38:357-368.

Rumpel S, Hatt H, Gottmann K (1998) Silent synapses in the developing rat visual cortex: evidence for postsynaptic expression of synaptic plasticity. J Neurosci 18:8863-8874.

Ruthazer ES, Akerman CJ, Cline HT (2003) Control of axon branch dynamics by correlated activity in vivo. Science 301:66-70.

Ruthazer ES, Li J, Cline HT (2006) Stabilization of axon branch dynamics by synaptic maturation. J Neurosci 26:3594-3603.

Shi J, Aamodt SM, Townsend M, Constantine-Paton M (2001) Developmental depression of glutamate neurotransmission by chronic low-level activation of NMDA receptors. J Neurosci 21:6233-6244.

Simon DK, Prusky GT, O'Leary DD, Constantine-Paton M (1992) $N$-Methyl-D-aspartate receptor antagonists disrupt the formation of a mammalian neural map. Proc Natl Acad Sci USA 89:10593-10597.

Sjostrom PJ, Hausser M (2006) A cooperative switch determines the sign of synaptic plasticity in distal dendrites of neocortical pyramidal neurons. Neuron 51:227-238.

Takahashi T, Svoboda K, Malinow R (2003) Experience strengthening transmission by driving AMPA receptors into synapses. Science 299:1585-1588.

Torborg CL, Feller MB (2005) Spontaneous patterned retinal activity and the refinement of retinal projections. Prog Neurobiol 76:213-235.
Torborg CL, Hansen KA, Feller MB (2005) High frequency, synchronized bursting drives eye-specific segregation of retinogeniculate projections. Nat Neurosci 8:72-78.

Tzounopoulos T, Kim Y, Oertel D, Trussell LO (2004) Cell-specific, spike timing-dependent plasticities in the dorsal cochlear nucleus. Nat Neurosci 7:719-725.

Wu G, Malinow R, Cline HT (1996) Maturation of a central glutamatergic synapse. Science 274:972-976.

Xu W, Orr-Urtreger A, Nigro F, Gelber S, Sutcliffe CB, Armstrong D, Patrick JW, Role LW, Beaudet AL, De Biasi M (1999) Multiorgan autonomic dysfunction in mice lacking the $\beta 2$ and the $\beta 4$ subunits of neuronal nicotinic acetylcholine receptors. J Neurosci 19:9298-9305.

Xu-Friedman MA, Regehr WG (1999) Presynaptic strontium dynamics and synaptic transmission. Biophys J 76:2029-2042.

Zhang LI, Poo MM (2001) Electrical activity and development of neural circuits. Nat Neurosci 4 [Suppl]:1207-1214.

Zhao JP, Constantine-Paton M (2004) Eye opening changes plasticity at visual synapses. Soc Neurosci Abstr 30:154.11.

Zhao JP, Phillips MA, Constantine-Paton M (2006) Long-term potentiation in the juvenile superior colliculus requires simultaneous activation of NMDA receptors and L-type $\mathrm{Ca}^{2+}$ channels and reflects addition of newly functional synapses. J Neurosci 26:12647-12655. 